\title{
Noncommutative Geometry and String Duality
}

\author{
F. Lizzi ${ }^{a *}$ and R.J. Szabo ${ }^{b}$ \\ ${ }^{a}$ Dipartimento di Scienze Fisiche, Università di Napoli Federico II \\ and INFN, Sezione di Napoli, Mostra d'Oltramare Pad. 20, 80125 Napoli, Italy \\ E-mail: if edele.lizzi@na.infn.itti \\ ${ }^{b}$ The Niels Bohr Institute \\ Blegdamsvej 17, DK-2100 Copenhagen Ø, Denmark \\ E-mail: 'szabo@nbi.dk'
}

ABSTRACT: A review of the applications of noncommutative geometry to a systematic formulation of duality symmetries in string theory is presented. The spectral triples associated with a lattice vertex operator algebra and the corresponding Dirac-Ramond operators are constructed and shown to naturally incorporate target space and discrete worldsheet dualities as isometries of the noncommutative space. The target space duality and diffeomorphism symmetries are shown to act as gauge transformations of the geometry. The connections with the noncommutative torus and Matrix Theory compactifications are also discussed.

\section{Introduction}

String theory in the previous decade raised some expectations about the nature of geometry at very small distance scales. Because strings have a finite intrinsic length scale $l_{s}$, it may not be possible to observe distances smaller than $l_{s}$. Thus if one uses only strings as probes of short distance structure, the conventional ideas of general relativity break down at lengths of the order of $l_{s}$. This is exemplified through string modified uncertainty relations lower bound on the measurability of lengths in the spacetime. The spacetime coordinates thus become smeared out and at short distances the notion of a "point" becomes meaningless.

Another piece of evidence is the $T$-duality symmetry of strings compactified on a circle $S^{1}$ of radius $R$ [2]. This is a quantum symmetry which maps the string theory onto one with target space the circle of dual radius $\tilde{R}=l_{s}^{2} / R$, and at the same time interchanges the momenta of the strings with their winding numbers around the $S^{1}$ in the spectrum of the quantum string theory. Because of this symmetry, the moduli

\footnotetext{
${ }^{*}$ Conference Speaker
}

space of string theories on $S^{1}$ is parametrized by radii $R \geq l_{s}$, and very small circles are unobservable since the corresponding string theory can be mapped onto a completely equivalent one living on a very large $S^{1}$. This leads to the notion of quantum geometry, defined to be the appropriate modification of classical general relativity implied by string theory.

The uncertainty relations tell us that spacetime at very small distances should be thought of as a quantum object. The appropriate mathematical arena for the study of such "pointless" geometry is the theory of algebras started by von Neumann, which in more modern times has developed into noncommutative geometry [3in]. Noncommutative geometry presents an alternative, algebraic approach to the study of Riemannian geometry and its generalizations, such as those hinted by string theory. In this paper we shall discuss the applications of noncommutative geometry towards a systematic development of the notion of quantum geometry.

Despite the implications on short distance structure presented by string theory, many questions have been answered using only classical geometry. This has occured in part because of the 
extremely rich mathematical structures embedded into string compactifications which enables one to develop to a great extent effective field theories on moduli spaces. However, the effective field theories hide the true internal symmetries of string theory, and to study the internal Kaluza-Klein spaces the notion of spacetime geometry needs a drastic modification. This has become especially clear over the last few years when it has been realized that the low energy effective field theory for D-branes in string theory has configuration space which is described in terms of non-commuting, matrix valued spacetime coordinate fields 蔔. This has led to, among other things, the Matrix Theory conjecture [i-in] which proposes a light-cone frame description of M-theory in terms of the Hamiltonian dynamics of D0-branes. This rich structure indicates that some sort of generalization of geometry is needed to describe the internal degrees of freedom implied by D-branes and M-theory, and indeed it has been shown recently that noncommutative geometry is the natural setting in which to study toroidal compactifications of Matrix Theory [6].

In this paper we shall review the formulation of quantum geometry through the techniques of noncommutative geometry, based mostly on the

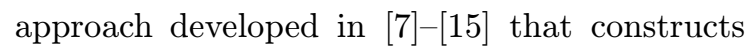
a "space" in which string duality is naturally realized as a true geometric symmetry. Starting from a brief review of the ideas from noncommutative geometry that we shall need, we shall describe the construction of the FröhlichGawędzki geometry [i] algebraic properties of vertex operator algebras. We will then describe how string duality naturally leads to the quantum geometry of classical spacetimes within this framework, and how the dualities manifest themselves as internal gauge symmetries of the noncommutative geometry

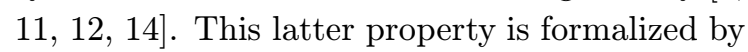
a remarkable connection between string geometry and the noncommutative torus [1 13 in, which also allows us to relate this worldsheet approach to the target space descriptions using Matrix Theory compactifications.

\section{Spectral Triples in Noncommuta- tive Geometry}

Noncommutative geometry is the study of geometric spaces (and their generalizations) using algebras of fields defined on them. In this article we shall discuss how to describe stringy spacetime as a noncommutative geometry, and how the symmetries of the theory (such as $T$-duality and spacetime diffeomorphisms) are realized as gauge transformations. The starting point is to discuss an algebraic framework for ordinary commutative geometry. Usually a compact Riemannian manifold $M$ is characterized as a topological space on which locally it is possible to introduce points $x \in M$ characterized by a finite number of real numbers $x^{i} \in \mathbb{R}$. Distances in $M$ are determined by the metric of the space,

$$
d s^{2}=g_{i j}(x) d x^{i} d x^{j}
$$

via the formula for geodesic length

$$
d(x, y)=\inf _{\gamma_{x, y}} \int_{\gamma_{x, y}} d s
$$

where $\gamma_{x, y}$ is a path from the point $x$ to the point $y$ in $M$.

There is a dual description of the topology and differentiable structure of a smooth manifold which is provided by the $*$-algebra $\mathcal{A}=$ $C^{\infty}(M, \mathbb{C})$ of smooth complex-valued functions $f: M \rightarrow \mathbb{C}$ (This algebra can be thought of as the algebra "generated" by the points of $M$ ). The completion of this algebra is the commutative $C^{*}$-algebra $C^{0}(M, \mathbb{C})$ of continuous complexvalued functions on $M$ with the $L^{\infty}$-norm

$$
\|f\|_{\infty}=\sup _{x \in M}|f(x)|
$$

The algebra $C^{0}(M, \mathbb{C})$ encodes all of the information about the topology of the space through the continuity criterion. Thus, in general, given a topological space one may naturally associate to it an abelian $C^{*}$-algebra. That the converse is also true is known as the Gel'fand-Naimark theorem [1] ${ }_{1}^{6}$. Namely, there is an isomorphism between the category of Hausdorff topological spaces $M$ and the category of commutative $C^{*}$ algebras $\mathcal{A}$. The Gel'fand-Naimark functor is constructed by using the fact that given an abelian 
$C^{*}$-algebra $\mathcal{A}$, it is possible to reconstruct a topological space $M$ as the structure space of characters of the algebra, i.e. the $*$-linear multiplicative functionals $\chi: \mathcal{A} \rightarrow \mathbb{C}$. Points $x \in M$ are then obtained via the identification

$$
\chi_{x}(f)=f(x) \quad, \quad \forall f \in \mathcal{A}
$$

and the topology is obtained in an unambiguous way from the notion of pointwise convergence (in the usual topology of $\mathbb{C}$ ). Note that for a commutative algebra a character is the same thing as an irreducible representation of $\mathcal{A}$.

What this all means is that the study of the properties of topological spaces can be substituted by a purely algebraic description in terms of abelian $C^{*}$-algebras. A noncommutative space is then obtained by replacing $C^{0}(M, \mathbb{C})$ by some non-abelian $C^{*}$-algebra. In that case, not all irreducible representations of the algebra are onedimensional and the identification of "points" becomes ambiguous. But as we shall see, the purely algebraic approach of noncommutative geometry is particularly well-suited to describe the intrinsic symmetries of string theory. Note that the *-algebra $C^{\infty}(M, \mathbb{C})$ also encodes all of the information about the differentiable structure of a manifold $M$ through the smoothness criterion. In what follows it will suffice to have a description in terms of only a dense subalgebra of a given $C^{*}$-algebra.

The metric aspect and other geometrical properties are introduced into this framework by using the fact that any $C^{*}$-algebra can be represented faithfully and unitarily as a subalgebra of the algebra $\mathcal{B}(\mathcal{H})$ of bounded operators acting on some separable Hilbert space $\mathcal{H}\left[\overline{1}_{1}\right]$. In the following often we will not distinguish between the abstract algebra $\mathcal{A}$ and its representation $\pi(\mathcal{A})$ (the norm on $\mathcal{A}$ is thus always understood as the operator norm and the $*$-involution as Hermitian conjugation). An "infinitesimal length element" is introduced by the relation

$$
d s^{-1}=D
$$

where $D$ is a (not necessarily bounded) operator on $\mathcal{H}$ which is called a generalized Dirac operator [3i] and which satisfies the following properties:

$$
\text { - } \left.D=D^{\dagger} \text { (this ensures positivity } d s^{2} \geq 0\right)
$$

- $[D, f] \in \mathcal{B}(\mathcal{H}) \quad \forall f \in \mathcal{A}$

- $D$ has compact resolvent

A metric space structure is then given by the Connes distance function on the structure space of $\mathcal{A}$,

$$
d(x, y)=\sup _{f \in \mathcal{A}:\|[D, f]\| \leq 1}\left|\chi_{x}(f)-\chi_{y}(f)\right|
$$

The generalized Dirac operator also enables one to introduce concepts from ordinary differential geometry. Using $D$ one may define a representation of abstract differential one-forms as

$$
\pi_{D}(f d g)=f[D, g]
$$

and similarly one can introduce a representation of higher degree forms (In that case a quotient space must be considered in order to eliminate the so-called junk forms) [혹. Gauge theories are also readily generalized to this setting. Generally, within this algebraic framework a vector bundle corresponds to a finitely-generated projective module over the algebra $\mathcal{A}$ (the corresponding space of smooth sections when $\mathcal{A}=$ $\left.C^{\infty}(M, \mathbb{C})\right)$. However, in the following we shall consider, for simplicity, only the case of trivial bundles. A gauge potential $A$ is then a one-form of the type (2.7) and a connection is provided via the Dirac operator through the definition of a gauge covariant derivative

$$
D_{A}=D+A
$$

The gauge group is defined as the group of unitary elements of the algebra,

$$
\mathcal{U}(\mathcal{A})=\left\{u \in \mathcal{A} \mid u^{\dagger} u=u u^{\dagger}=\mathbb{I}\right\}
$$

and gauge transformations are the inner automorphisms of the algebra, i.e. the maps $g_{u}$ : $\mathcal{A} \rightarrow \mathcal{A}$ which act as conjugation by a unitary element $u \in \mathcal{U}(\mathcal{A})$,

$$
g_{u}(f)=u f u^{\dagger}
$$

The usual ingredients of a gauge theory on a manifold may then be summarized as the following algebraic elements:

- Connections: $A=\sum_{n} f_{n}\left[D, g_{n}\right], f_{n}, g_{n} \in$ $\mathcal{A}$ 
- Curvature: $F=[D, A]+A^{2}$

- Bosonic Action: $f F^{2}$ (where $f$ is a regularized trace, e.g. the Dixmier trace [3inj]

- Fermionic Action: $f \bar{\psi} D_{A} \psi$

The set of three ingredients $(\mathcal{A}, \mathcal{H}, D)$, i.e. a $*$-algebra $\mathcal{A}$ of bounded operators acting on a separable Hilbert space $\mathcal{H}$ and a generalized Dirac operator $D$ on $\mathcal{H}$, is called a spectral triple (or a Dirac K-cycle). The spectral triple encodes all the topological and geometrical information about a Riemannian manifold. But notice that its construction is made with no reference to any underlying space, and that it also applies to generic (not necessarily commutative) algebras. We shall now describe a number of examples.

\section{Spin Manifolds}

If $M$ is a compact spin manifold, we take

$$
\begin{aligned}
\mathcal{A} & =C^{\infty}(M, \mathbb{C}) \\
\mathcal{H} & =L^{2}(M, S) \\
D & =i \gamma^{i} \nabla_{i}
\end{aligned}
$$

where $L^{2}(M, S)$ is the Hilbert space of square integrable spinors on $M$ and the algebra $\mathcal{A}$ acts diagonally on $\mathcal{H}$ by pointwise multiplication. Here $\gamma^{i}$ are the usual Dirac matrices generating the Clifford algebra $\left\{\gamma^{i}, \gamma^{j}\right\}=2 g^{i j}$ of $M$ and $\nabla=$ $d+\Gamma$ is the usual covariant derivative constructed from the spin connection of $M$. The invariant line element of $M$ is now represented as the free massless fermion propagator on $M$ and the distance function $(2.6 i)$ is

$$
d(x, y)=\sup _{|\nabla f| \leq 1}|f(x)-f(y)|
$$

Note that the distance formula ( $(\overline{2} . \overline{1} \overline{2})$, which is defined in terms of complex-valued functions on $M$, is dual to the geodesic distance formula $(2.27)$, which is defined in terms of arcs connecting $x$ to $y$ in $M$. Notice also how the Riemannian geometry of $M$ is naturally encoded within the definition of the Dirac operator.

The action functionals described above corresponding to a gauge theory constructed from this spectral triple yield the usual one for electrodynamics on the manifold $M$, with gauge group the unimodular loop group $C^{\infty}\left(M, S^{1}\right)$ of $U(1)$ gauge transformations on $M$. Note that the spectral triple $\left(\overline{2} . \overline{1} \overline{1}_{1}^{\prime}\right)$ is that which naturally arises from quantizing the free geodesic motion of a test particle on $M$. Then $\mathcal{A}$ is the algebra of observables, $\mathcal{H}$ is the Hilbert space of physical states, and the Hamiltonian $H=-D^{2}$ is the LaplaceBeltrami operator of $M$. Thus ordinary (commutative) spaces can be thought of as those probed by quantum mechanical test particles. It is in this way that noncommutative geometry may be thought of as "quantum geometry".

\section{Morita Equivalence}

The natural extension of the previous example is

$$
\begin{aligned}
\mathcal{A} & =C^{\infty}(M, \mathbb{C}) \otimes M_{N}(\mathbb{C}) \\
\mathcal{H} & =L^{2}(M, S)^{\oplus N} \\
D & =i \gamma^{i} \nabla_{i} \otimes I_{N}
\end{aligned}
$$

where $M_{N}(\mathbb{C})$ is the algebra of $N \times N$ complexvalued matrices and $I_{N}$ is the $N \times N$ identity matrix. The gauge group is now the group of $U(N)$ gauge transformations $C^{\infty}(M, U(N))$ on $M$ and the spinors of $\mathcal{H}$ transform in the vector representation of $U(N)$. Since the algebra $\mathcal{A}$ of matrix-valued functions on the manifold $M$ is noncommutative, the Gel'fand-Naimark theorem does not apply to this case and it is not possible to formally identify "points" of a space. In fact, there is an $N$ dimensional sphere of pure states at each point (corresponding to the various unitary equivalent representations) which can be thought of as an internal Kaluza-Klein isospin space with points connected by $U(N)$ gauge transformations. Nevertheless, it is clear that the configuration space of the quantum theory corresponding to $\left(\overline{2} . \overline{1} \overline{3}_{1}\right)$ is still the manifold $M$. The choices of abelian subalgebras of $M_{N}(\mathbb{C})$ create $N$ copies of the same manifold connected by gauge transformation.

The apparent paradox is resolved by noticing that the algebra $M_{N}(\mathbb{C})$ has only one non-trivial irreducible representation as a $C^{*}$-algebra. Thus

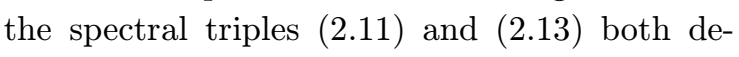
termine the same space. This phenomenon is captured formally by saying that the algebras $C^{\infty}(M, \mathbb{C})$ and $C^{\infty}(M, \mathbb{C}) \otimes M_{N}(\mathbb{C})$ are Morita equivalent. A $C^{*}$-algebra $\mathcal{B}$ is Morita equivalent 
to a $C^{*}$-algebra $\mathcal{A}$ if it is isomorphic to the algebra $\operatorname{End}_{\mathcal{A}}^{0}(\mathcal{E})$ of compact endomorphisms of some $\mathcal{A}$-module $\mathcal{E}$. This means that the two algebras become isomorphic upon tensoring them with the algebra of compact operators. Morita equivalent $C^{*}$-algebras have equivalent representation theories. They therefore differ only in the structure of their internal spaces (i.e. their gauge symmetries). The action functionals corresponding to $\left(12 . \overline{1} 3_{1}\right)$ yield the usual massless $U(N)$ gauge theory on the manifold $M$.

\section{The Two-sheeted Spacetime}

Let us now consider the spectral triple [i]

$$
\begin{aligned}
\mathcal{A} & =C^{\infty}(M, \mathbb{C}) \otimes \mathbb{Z}_{2} \\
\mathcal{H} & =L^{2}(M, S) \otimes \mathbb{Z}_{2} \\
D & =\left(\begin{array}{cc}
i \gamma^{i} \nabla_{i} & m \\
m^{*} & i \gamma^{i} \nabla_{i}
\end{array}\right)
\end{aligned}
$$

where $m$ is a fermion mass. The bosonic action functional corresponding to $(\overline{2} . \overline{1} \overline{4})$ gives not only the usual Yang-Mills term, but also the Higgs potential with its biquadratic form. This (commutative) space therefore gives a geometrical origin for the Higgs mechanism associated with the spontaneous breaking of the $U(1) \times U(1)$ gauge symmetry of $\mathcal{A}$ down to $U(1)$ corresponding to the diagonal projection of $\mathcal{A}$ onto $C^{\infty}(M, \mathbb{C})$.

\section{The Standard Model}

The previous example can be generalized to the noncommutative geometry $[\overline{1}[\overline{1}]$

$$
\begin{aligned}
\mathcal{A} & =C^{\infty}(M, \mathbb{C}) \otimes\left[\mathbb{C} \oplus \mathbb{H} \oplus M_{3}(\mathbb{C})\right] \\
\mathcal{H} & =L^{2}(M, S) \otimes\left[\mathbb{C} \oplus \mathbb{C}^{2} \oplus \mathbb{C}^{3}\right] \\
D & =\left(\begin{array}{cc}
i \gamma^{i} \nabla_{i} \otimes I_{3} & \mathcal{M} \\
\mathcal{M}^{\dagger} & i \gamma^{i} \nabla_{i} \otimes I_{3}
\end{array}\right)
\end{aligned}
$$

where $\mathbb{H}$ is the algebra of quaternions and $\mathcal{M}$ is the $3 \times 3$ fermion mass matrix. The unimodular group of the algebra $\mathcal{A}$ is the familiar gauge group $C^{\infty}(M, U(1) \times S U(2) \times S U(3))$ of the standard model and $\mathcal{H}$ represents the physical Hilbert space of six generation fermions. The action functional consists of the Yang-Mills action and the Higgs term. The spectral triple ( $2 . \overline{1} \overline{1})$ thereby shows how electroweak and chromodynamic degrees of freedom are induced by the geometry involving a discrete internal Kaluza-Klein space.

\section{The Noncommutative Torus}

The spectral triples we have considered thus far all have the property that they contain an underlying ordinary geometry, i.e. their algebras have the form $\mathcal{A}=C^{\infty}(M, \mathbb{C}) \otimes \mathcal{A}_{F}$ where $\mathcal{A}_{F}$ is a finite dimensional algebra. We now turn to a genuine example of a noncommutative geometry, in which it is impossible to think of "points", that will turn out to play a prominant role in the string theory applications.

Consider a $d$ dimensional torus $T_{d}=\mathbb{R}^{d} / 2 \pi \Gamma$, where $\Gamma$ is a Euclidean lattice of rank $d$ with bilinear form $g_{i j}$. Let $\omega^{i j}$ be a real-valued antisymmetric matrix. We define an algebra $\mathcal{A}^{(\omega)}$ with generators $U_{i}, i=1, \ldots, d$, and relations

$$
\begin{aligned}
& U_{i} U_{i}^{\dagger}=U_{i}^{\dagger} U_{i}=\mathbb{I} \\
& U_{i} U_{j}=\mathrm{e}^{2 \pi i \omega^{i j}} U_{j} U_{i}
\end{aligned}
$$

A generic "smooth" element $f$ of the completion $\mathcal{A}_{\infty}^{(\omega)}$ of $\mathcal{A}^{(\omega)}$ is a linear combination of monomials in the $U_{i}$,

$$
f=\sum_{p \in \Gamma^{*}} f_{p} U_{1}^{p_{1}} U_{2}^{p_{2}} \cdots U_{d}^{p_{d}}
$$

where $\Gamma^{*}$ is the dual lattice to $\Gamma$ and $f_{p}$ are elements of the Schwartz space $\mathcal{S}\left(\Gamma^{*}\right)$ of sequences of rapid decrease. The abstract algebra $\mathcal{A}_{\infty}^{(\omega)}$ can be given a concrete representation as a quantum deformation of the algebra $C^{\infty}\left(T_{d}, \mathbb{C}\right)$ of functions on the torus. The product of two functions $f, g \in C^{\infty}\left(T_{d}, \mathbb{C}\right)$ is now given by

$$
\left(f \star_{\omega} g\right)(x)=\left.\exp \left(i \pi \omega^{i j} \frac{\partial}{\partial x^{i}} \frac{\partial}{\partial x^{\prime j}}\right) f(x) g\left(x^{\prime}\right)\right|_{x^{\prime}=x}
$$

which is just the usual rule for multiplying Weyl ordered symbols of quantum mechanical operators. With the product (1.2.1-1) the generators in $\left(\overline{2} . \overline{1} \overline{6}_{1}\right)$ are just the usual basic plane waves

$$
U_{i}=\mathrm{e}^{i x^{i}}
$$

and the expansion $\left(\overline{2} . \overline{1} \bar{z}_{1}\right)$ can be thought of as a generalized Fourier series expansion.

It follows that $\mathcal{A}_{\infty}^{(0)} \cong C^{\infty}\left(T_{d}, \mathbb{C}\right)$. For $\omega^{i j} \neq$ 0 , the algebra $\mathcal{A}_{\infty}^{(\omega)}$ represents the quotient of the ordinary torus by the orbit of a free particle in it whose velocity vector forms an angle $\omega^{i j}$ with respect to cycles $i$ and $j$ of $T_{d}$. When the $\omega^{i j}$ are 
all rational numbers, the algebra $\mathcal{A}_{\infty}^{(\omega)}$ is therefore Morita equivalent to $C^{\infty}\left(T_{d}, \mathbb{C}\right)$. When the $\omega^{i j}$ are irrational, the orbits are dense and ergodic and the resulting quotient space is not a conventional Hausdorff manifold. In any of the cases, an invariant integration may be introduced via the unique trace $f: \mathcal{A}_{\infty}^{(\omega)} \rightarrow \mathbb{C}$ which is given by the classical average

$$
f f=\int_{T_{d}} \prod_{i=1}^{d} \frac{d x^{i}}{2 \pi} f(x)=f_{0}
$$

and a Dirac operator may be introduced via the natural set of linear derivations $\Delta_{i}: \mathcal{A}_{\infty}^{(\omega)} \rightarrow$ $\mathcal{A}_{\infty}^{(\omega)}, i=1, \ldots, d$, defined by the logarithmic derivatives

$$
\Delta_{i}\left(U_{j}\right)=\delta_{i j} U_{j}
$$

One aspect of this example which will be particularly important for us is the set of Morita equivalence classes of noncommutative tori [1 $\left.1 \overline{8}^{\prime}\right]$. From ('2.1-i') it follows that the noncommutative tori with deformation parameters $\omega^{i j}$ and $\omega^{i j}+$ $\lambda^{i j}$, with $\lambda^{i j}$ any antisymmetric integer valued matrix, are the same. This symmetry is part of a larger group $O(d, d ; \mathbb{Z})$ which parametrizes the Morita equivalence classes. It acts naturally on the deformation matrix $\omega$ as (upon picking a suitable basis of $\mathbb{R}^{d, d}$ )

$$
\begin{array}{r}
\omega \rightarrow \omega^{*}=(A \omega+B)(C \omega+D)^{-1} \\
\text { with }\left(\begin{array}{ll}
A & B \\
C & D
\end{array}\right) \in O(d, d ; \mathbb{Z})
\end{array}
$$

where $A, B, C, D$ are $d \times d$ integer valued matrices which satisfy the relations

$$
\begin{aligned}
& A^{\top} C+C^{\top} A=0=B^{\top} D+D^{\top} B \\
& A^{\top} D+C^{\top} B=I_{d}
\end{aligned}
$$

Later on we will see that for toroidally compactified string theory, Morita equivalence of the corresponding noncommutative tori is precisely the same notion as target space duality on the associated toroidal string background.

\section{Noncommutative String Spacetimes}

We now seek some sort of algebra which describes the "noncommutative coordinates" of spacetime as seen by strings. The structure should be such that at very large distance scales (much larger than $l_{s}$ ), where the strings effectively become point particles which are well described by ordinary quantum field theory, we recover a usual (commutative) spacetime manifold $M$ as described in section 2. The elegant proposal of Fröhlich

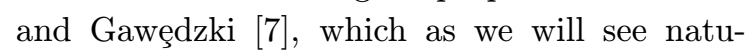
rally incorporates duality as a gauge symmetry of the corresponding spectral triple, is to take $\mathcal{A}$ to be the vertex operator algebra of the underlying worldsheet conformal field theory of the string theory. Vertex operators describe interactions of strings and they operate on the string Hilbert space as insertions on the worldsheet corresponding to the emission or absorption of string states. They therefore form the appropriate noncommutative algebra which describes the quantum geometry of the "space" of interacting strings. In this section we shall construct the spectral triple associated with a toroidally compactified bosonic string theory. The theory of vertex operator alge-

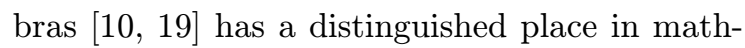
ematics (having connections with the theory of modular functions, the Monster sporadic group, etc.). We shall start by giving a very brief general overview of the definition and properties of a vertex operator algebra.

\section{Vertex Operator Algebras}

Any conformal field theory naturally has associated to it two chiral algebras $\mathcal{E}^{ \pm}$which form the operator product algebras of the (anti-)holomorphic fields of the theory. They contain two mutually commuting representations of the infinite dimensional Virasoro algebra (generating the conformal invariance of the theory)

$$
\left[L_{k}^{ \pm}, L_{m}^{ \pm}\right]=(k-m) L_{k+m}^{ \pm}+\frac{c}{12}\left(k^{3}-k\right) \delta_{k+m, 0}
$$

where $c$ is the central charge of the string theory. The algebras $\mathcal{E}^{ \pm}$act densely on the Hilbert spaces $\mathcal{H}^{ \pm}$, of left and right handed string states respectively. The vertex operator algebra is constructed using the operator-state correspondence of local quantum field theory. Namely, to each state $\psi^{( \pm)} \in \mathcal{H}^{ \pm}$there corresponds a chiral vertex operator $V_{ \pm}\left(\psi^{( \pm)} ; z_{ \pm}\right)$, where $\left(z_{+}, z_{-}\right)$are local coordinates on a Riemann surface (here we as- 
sume that the worldsheet is the Riemann sphere). For a bosonic string theory, the vertex operators can be expanded as Laurent series

$$
V_{ \pm}\left(\psi^{( \pm)} ; z_{ \pm}\right)=\sum_{n \in \mathbb{Z}} \psi_{n}^{( \pm)} z_{ \pm}^{-n-1}
$$

The vertex operator algebra $\mathcal{A}$ is now characterized by some algebraic relations which can be summarized as follows. The commutation relations are determined by the braiding relations

$$
\begin{aligned}
& {\left[V_{ \pm}\left(\psi_{I}^{( \pm)} ; z_{ \pm}\right) V_{ \pm}\left(\psi_{J}^{( \pm)} ; w_{ \pm}\right)\right]_{\gamma_{z, w}^{ \pm}}} \\
& =\sum_{K, L}\left(R^{ \pm}\right)_{I J}^{K L} V_{ \pm}\left(\psi_{K}^{( \pm)} ; w_{ \pm}\right) V_{ \pm}\left(\psi_{L}^{( \pm)} ; z_{ \pm}\right)
\end{aligned}
$$

where $R^{ \pm}$are called braiding matrices and $[\cdot]_{\gamma_{z, w}^{ \pm}}$ is the operator which exchanges the two points $z_{ \pm} \leftrightarrow w_{ \pm}$along a path $\gamma_{z, w}^{ \pm}$with (anti-)clockwise orientation on the worldsheet. The products in $\left(3.3^{i}\right)$ are well defined operators on $\mathcal{H}^{ \pm}$provided that $\left|z_{ \pm}\right|<\left|w_{ \pm}\right|$. Furthermore, chiral vertex operators may be composed together using the fusion equations

$$
\begin{aligned}
& V_{ \pm}\left(\psi_{I}^{( \pm)} ; z_{ \pm}\right) V_{ \pm}\left(\psi_{J}^{( \pm)} ; w_{ \pm}\right)=\sum_{K, L}\left(F^{ \pm}\right)_{I J}^{K L} \\
& \times V_{ \pm}\left(V_{ \pm}\left(\psi_{K}^{( \pm)} ; z_{ \pm}-w_{ \pm}\right) \psi_{L}^{( \pm)} ; w_{ \pm}\right)
\end{aligned}
$$

where $F^{ \pm}$are called fusion matrices. The braiding and fusion relations completely characterize the chiral algebras $\mathcal{E}^{ \pm}$. They can be combined into a single relation known as the Jacobi identity of the vertex operator algebra [1] ${ }^{1} \overline{9}_{1}^{\prime}$, which can be thought of as a combination of the classical Jacobi identity for Lie algebras and the Cauchy residue formula for meromorphic functions.

The full, left-right symmetric vertex operator algebra is now obtained via the sewing transformations

$$
\begin{aligned}
& V\left(\psi_{I} ; z_{+}, z_{-}\right) \\
& =\sum_{K, L} D_{I}^{K L} V_{+}\left(\psi_{K}^{(+)} ; z_{+}\right) \otimes V_{-}\left(\psi_{L}^{(-)} ; z_{-}\right)
\end{aligned}
$$

where $D_{I}^{K L}$ are complex valued sewing coefficients and $\psi_{I}=\psi_{I}^{(+)} \otimes \psi_{I}^{(-)}$. The local fields $(3.5)$ act as operator valued distributions on the
Hilbert space $\mathcal{H}=\mathbb{C}^{\{D\}} \otimes \mathcal{H}^{+} \otimes \mathcal{H}^{-}$, where $\mathbb{C}^{\{D\}}$ is the finite dimensional multiplicity space which labels the various left-right sewings. The operator-state correspondence is represented by the relation $V(\psi ; 0,0) \mid$ vac $\rangle=\psi \in \mathcal{H}$, where $\mid$ vac $\rangle$ is the vacuum state of $\mathcal{H}$ (which we assume is unique). Locality is the constraint that two operators of the type (3.5i) commute whenever their worldsheet arguments are space-like separated. From this constraint it is possible to actually combine the braiding and fusion relations into a reduced set of relations known as the operator product expansion of two local conformal fields $(3.5)$. For this, we grade the Hilbert space $\mathcal{H}$ by conformal dimensions $\Delta_{I}^{ \pm}$which are the highest weights of the representations of the Virasoro algebra in $\mathcal{E}^{ \pm}$. The corresponding highest weight vectors $\psi_{I}$ for $\mathcal{E}^{+} \otimes \mathcal{E}^{-}$are called primary states and are defined by

$$
L_{0}^{ \pm} \psi_{I}=\Delta_{I}^{ \pm} \psi_{I} \quad, \quad L_{k}^{ \pm} \psi_{I}=0 \quad \forall k>0
$$

The associated vertex operators are called primary fields and they satisfy the differential equations

$$
\begin{aligned}
& {\left[L_{k}^{ \pm}, V\left(\psi_{I} ; z_{+}, z_{-}\right)\right]} \\
& =\left(z_{ \pm}^{k+1} \frac{\partial}{\partial z_{ \pm}}+(k+1) \Delta_{I}^{ \pm} z_{ \pm}^{k}\right) V\left(\psi_{I} ; z_{+}, z_{-}\right)
\end{aligned}
$$

With a suitable normalization of the two-point functions of primary fields of fixed conformal dimension, one may derive the operator product expansion

$$
\begin{aligned}
& V\left(\psi_{I} ; z_{+}, z_{-}\right) V\left(\psi_{J} ; w_{+}, w_{-}\right) \\
& =\sum_{K} C_{I J K}\left(z_{+}-w_{+}\right)^{\Delta_{K}^{+}-\Delta_{I}^{+}-\Delta_{J}^{+}} \\
& \quad \times\left(z_{-}-w_{-}\right)^{\Delta_{K}^{-}-\Delta_{I}^{-}-\Delta_{J}^{-}} V\left(\psi_{K} ; z_{+}, z_{-}\right)
\end{aligned}
$$

where the sum runs over a complete set of primary fields (equivalently orthonormal primary states $\psi_{K} \in \mathcal{H}$ ), and $C_{I J K}$ are the constant operator product expansion coefficients which are functions of the braiding, fusion and sewing coefficients introduced above. The relation (3.8) completely characterizes the vertex operator algebra, which as we see is a rather complicated unital $*$-algebra. 


\section{Lattice Vertex Operator Algebras}

We shall now specialize the above discussion to the case of closed bosonic strings propagating in a $d$-dimensional toroidal target space $T_{d}=$ $\mathbb{R}^{d} / 2 \pi \Gamma$. The classical string embedding fields in such a target space are determined as the mod $2 \pi \Gamma$ periodic solutions of the two dimensional wave equation, which are given by the chiral multivalued Fubini-Veneziano fields (in units with $\left.l_{s}=1\right)$

$$
X_{ \pm}^{i}\left(z_{ \pm}\right)=x_{ \pm}^{i}+i g^{i j} p_{j}^{ \pm} \log z_{ \pm}+\sum_{k \neq 0} \frac{1}{i k} \alpha_{k}^{( \pm) i} z_{ \pm}^{-k}
$$

where $g^{i j}$ is the matrix inverse of the metric $g_{i j}$ of $T_{d},\left(\alpha_{k}^{( \pm) i}\right)^{*}=\alpha_{-k}^{( \pm) i}$ and $z_{ \pm}=\mathrm{e}^{-i(\tau \pm \sigma)}$ with $(\tau, \sigma)$ local coordinates on the cylinder $\mathbb{R} \times S^{1}$. The left-right momenta are given by

$$
p_{i}^{ \pm}=\frac{1}{\sqrt{2}}\left(p_{i} \pm d_{i j}^{ \pm} w^{j}\right)
$$

and the background matrices are

$$
d_{i j}^{ \pm}=g_{i j} \pm \beta_{i j}
$$

with $\beta_{i j}$ the antisymmetric constant torsion form of the target space. The zero modes $x^{i}=\frac{1}{\sqrt{2}}\left(x_{+}^{i}+\right.$ $\left.x_{-}^{i}\right) \in T_{d}$ represent the position of the center of mass of the string while $p_{i} \in \Gamma^{*}$ are the corresponding momenta. The $\alpha$ 's represent the vibrational modes of the string and $w^{i} \in \Gamma$ are the winding numbers which represent the number of times that the string wraps around the cycles of the torus. The set of momenta $\left(p^{+}, p^{-}\right)$live in the even, self-dual Lorentzian lattice $\Gamma^{*} \oplus \Gamma$.

Canonical quantization of this theory identifies the non-vanishing quantum commutators

$$
\begin{aligned}
{\left[p_{i}^{ \pm}, x_{ \pm}^{j}\right] } & =i \delta_{i}^{j} \\
{\left[\alpha_{k}^{( \pm) i}, \alpha_{m}^{( \pm) j}\right] } & =k g^{i j} \delta_{k+m, 0}
\end{aligned}
$$

The quantum fields $(\underline{3} . \overline{9} \cdot \overline{1})$ therefore act on the Hilbert space

$$
\mathcal{H}=L^{2}\left(T_{d} \times T_{d}^{*}, S\right) \otimes \mathcal{F}^{+} \otimes \mathcal{F}^{-}
$$

where $T_{d}^{*}=\mathbb{R}^{d} / 2 \pi \Gamma^{*}$ is the dual torus to $T_{d}$ and $S \rightarrow T_{d} \times T_{d}^{*}$ is the spin bundle over the double torus constructed from the self-dual lattice $\Gamma^{*} \oplus$ $\Gamma$. The $L^{2}$ space in $(13 . \overline{1}-1)$ is constructed from the zero mode operators and is spanned by the plane wave states

$$
\left|q^{+}, q^{-}\right\rangle=\mathrm{e}^{-i\left(q_{i}-\beta_{i j} v^{j}\right) x^{i}-i v^{i} x_{i}^{*}}
$$

where $x_{i}^{*}=\frac{1}{\sqrt{2}} g_{i j}\left(x_{+}^{j}+x_{-}^{j}\right) \in T_{d}^{*}$. The $\mathcal{F}^{ \pm}$are bosonic Fock spaces generated by the oscillatory modes $\alpha_{k}^{( \pm) i}$, respectively, and the unique vacuum state of $\left(\underline{\underline{3}} . \underline{1}_{-}^{\prime}\right)$ is $|\mathrm{vac}\rangle=|0,0\rangle \otimes|0\rangle_{+} \otimes|0\rangle_{-}$.

A vertex operator algebra for the toroidal compactification may now be constructed using the operator-state correspondence, as described above. The Hilbert space $(\bar{B} \cdot \overline{1} \cdot \overline{1})$ is spanned by states of the form

$$
\begin{gathered}
\psi=\left|q^{+}, q^{-}\right\rangle \otimes \prod_{a=1}^{N} r_{i}^{(a)+} \alpha_{-n_{a}}^{(+) i}|0\rangle_{+} \\
\otimes \prod_{b=1}^{M} r_{j}^{(b)-} \alpha_{-m_{b}}^{(-) j}|0\rangle_{-}
\end{gathered}
$$

where $\left(q^{+}, q^{-}\right),\left(r^{(a)+}, r^{(a)-}\right) \in \Gamma^{*} \oplus \Gamma$ and $n_{a}, m_{b}>$ 0 . To $\left(3.1-1 \overline{1}^{\prime}\right)$ we associate the vertex operator

$$
\begin{aligned}
& V\left(\psi ; z_{+}, z_{-}\right) \\
& ={ }_{\circ}^{\circ} i V_{q^{+} q^{-}}\left(z_{+}, z_{-}\right) \prod_{a=1}^{N} \frac{r_{i}^{(a)+}}{\left(n_{a}-1\right) !} \frac{d^{n_{a}} X_{+}^{i}\left(z_{+}\right)}{d z_{+}^{n_{a}}} \\
& \quad \times \prod_{b=1}^{M} \frac{r_{j}^{(b)-}}{\left(m_{b}-1\right) !} \frac{d^{m_{b}} X_{-}^{j}\left(z_{-}\right)}{d z_{-}^{m_{b}}} \circ
\end{aligned}
$$

where $\stackrel{\circ}{\circ} \cdot \stackrel{\circ}{\circ}$ denotes the usual Wick normal ordering of operators, and

$$
\begin{aligned}
& V_{q^{+} q^{-}}\left(z_{+}, z_{-}\right) \\
& =(-1)^{q_{i} w^{i}} \stackrel{\circ}{\circ} \mathrm{e}^{-i q_{i}^{+} X_{+}^{i}\left(z_{+}\right)-i q_{i}^{-} X_{-}^{i}\left(z_{-}\right)} \circ
\end{aligned}
$$

are the basic, left-right symmetric tachyon vertex operators which generate a unital $*$-algebra $\mathcal{A}$ which acts diagonally on (i. $\overline{1} \overline{1} \overline{1})$. The operatorvalued cocycle phases in $\left(\underline{3} .17^{\prime}\right)$ are inserted to give the vertex operators the correct locality relations. The algebraic properties of $\mathcal{A}$ are described by the operator product expansion, which for the tachyon generators reads

$$
\begin{aligned}
& V_{q^{+} q^{-}}\left(z_{+}, z_{-}\right) V_{r^{+} r^{-}}\left(w_{+}, w_{-}\right) \\
& =\mathrm{e}^{-\pi i\langle q, r\rangle} V_{r^{+} r^{-}}\left(w_{+}, w_{-}\right) V_{q^{+} q^{-}}\left(z_{+}, z_{-}\right)
\end{aligned}
$$


where we have assumed that $\pm \arg z_{ \pm}> \pm \arg w_{ \pm}$ and

$$
\langle q, r\rangle=q_{i}^{+} g^{i j} r_{j}^{+}-q_{i}^{-} g^{i j} r_{j}^{-}
$$

is the bilinear form on $\Gamma^{*} \oplus \Gamma$. We shall discuss this commutation relation in more detail later on. Note that the tachyon operators create the states $\left|q^{+}, q^{-}\right\rangle \in L^{2}\left(T_{d} \times T_{d}^{*}, S\right)$ in which all vibrational modes of the string are absent, i.e. they correspond to low energy states of the spacetime, which as we will see lead to ordinary (commutative) manifolds. On the other hand, the lowest symmetric stringy excitation of commutative spacetime is the state $\left|q^{+}, q^{-}\right\rangle \otimes \alpha_{-1}^{(+) i}|0\rangle_{+} \otimes$ $\alpha_{-1}^{(-) j}|0\rangle_{-}$which is created by the graviton operator

$$
\begin{aligned}
& V_{q^{+} q^{-}}^{i j}\left(z_{+}, z_{-}\right) \\
& ={ }_{\circ}^{\circ} i V_{q^{+} q^{-}}\left(z_{+}, z_{-}\right) \frac{d X_{+}^{i}\left(z_{+}\right)}{d z_{+}} \frac{d X_{-}^{j}\left(z_{-}\right)}{d z_{-}} \circ
\end{aligned}
$$

and represents the Fourier modes of the background matrices $d_{i j}^{ \pm}$.

\section{Spectral Triples for Toroidal Compactifi- cations}

The final object we require to complete the string theory spectral triple is an appropriate Dirac operator. In addition to the basic conformal symmetry of the model (generated by reparametrizations of the worldsheet coordinates), there is the target space reparametrization symmetry $X_{ \pm}^{i}\left(z_{ \pm}\right) \rightarrow$ $X_{ \pm}^{i}\left(z_{ \pm}\right)+\delta X_{ \pm}^{i}\left(z_{ \pm}\right)$, with $\delta X_{ \pm}^{i}\left(z_{ \pm}\right)$arbitrary periodic functions on $T_{d}$, which is generated by the conserved currents

$$
\delta_{ \pm}^{i}\left(z_{ \pm}\right)=-i z_{ \pm} \frac{d X_{ \pm}^{i}\left(z_{ \pm}\right)}{d z_{ \pm}}
$$

The operators $\left(\hat{3} . \overline{2} \overline{1_{-}^{\prime}}\right)$ generate a $u(1)_{+}^{d} \oplus u(1)_{-}^{d}$ Kac-Moody algebra whose highest weight states are the tachyon vectors described above. The spin bundle $S \rightarrow T_{d} \times T_{d}^{*}$ inherits a natural chirality grading from the corresponding Clifford module over the double torus, which yields two sets of Dirac matrices $\gamma_{i}^{ \pm}$that generate the Clifford algebra $\left\{\gamma_{i}^{ \pm}, \gamma_{j}^{ \pm}\right\}= \pm 2 g_{i j}$ (with all other anticommutators vanishing). This grading naturally splits the Hilbert space ( $(\overline{3} . \overline{1} \overline{1})$ as $\mathcal{H}=\mathcal{H}^{+} \oplus \mathcal{H}^{-}$ into the \pm 1 eigenspaces of the corresponding chirality operator.

The chiral structure of the theory now enables us to introduce two independent Dirac operators for the noncommutative geometry by

$$
D^{ \pm}\left(z_{ \pm}\right)=\gamma_{i}^{ \pm} \delta_{ \pm}^{i}\left(z_{ \pm}\right)=\sum_{k \in \mathbb{Z}} \gamma_{i}^{ \pm} \alpha_{k}^{( \pm) i} z_{ \pm}^{-k}
$$

where $\alpha_{0}^{( \pm) i}=g^{i j} p_{j}^{ \pm}$. The choices $\left(\underline{3}^{3} . \overline{2} \overline{2}^{i}\right)$ are not as ad-hoc as they may first seem. First of all, the operator $\delta_{ \pm}$generates reparametrizations of the target space, so that the first equality in ( $(3 . \overline{3} . \overline{2})$ is just the usual relationship between the Dirac operator and a covariant "derivative". Secondly, it is possible to show that the energy-momentum tensor of the conformal field theory is related to the Dirac operator by

$$
T^{ \pm}\left(z_{ \pm}\right)=\sum_{k \in \mathbb{Z}} L_{k}^{ \pm} z_{ \pm}^{-k-2}=-{ }_{\circ}^{\circ} D^{ \pm}\left(z_{ \pm}\right)^{2} \circ
$$

so that the square of the Dirac operator is related to the Hamiltonian in the same way as in the case of a spin-manifold. It can therefore be thought as generating the appropriate "LaplaceBeltrami operator" for the Riemannian geometry. Finally, it can also be shown that (i3..25) are the Ramond sector fermionic zero modes of the $N=1$ worldsheet supercharges which generate supersymmetry transformations of the appropriate supersymmetrization of the underlying worldsheet conformal sigma model. The quantized fermionic zero modes of these supercharges, when acting on states of vanishing total spacetime momentum, generate the deRham complex of the manifold $T_{d}$ - the two operators can be identified with the exterior derivative and coderivative, while harmonic forms correspond to supersymmetric (physical) states. This is just the classic Witten complex associated with twodimensional $N=1$ supersymmetric sigma models [200 deed appropriate to the quantum geometry associated with the string theory. The existence of two independent Dirac operators means that there are two spectral triples that may be constructed. We shall see that this feature severely restricts the effective spacetime geometry and is 
ultimately responsible for the occurence of duality symmetries in the quantum geometry.

\section{Target Space Duality}

As we will now show, the existence of two natural Dirac operators for the noncommutative geometry of the previous section is not an ambiguous property and is directly tied with the notion of duality. The main feature is that there are several isometries of the spectral triple that relate the chirally symmetric and antisymmetric Dirac operators $D, \bar{D}=D^{+} \pm D^{-}$. An isometry in the present context is a unitary operator $T: \mathcal{H} \rightarrow \mathcal{H}$ which is an automorphism of the vertex operator algebra $\mathcal{A}$, i.e. $T \mathcal{A} T^{-1}=\mathcal{A}$ (and in particular it preserves the operator-state correspondence), and which relates the two Dirac operators via

$$
D T=T \bar{D}
$$

This implies that, at the level of their spectral triples, the two spacetimes associated with $D$ and $\bar{D}$ are the same,

$$
(\mathcal{A}, \mathcal{H}, D) \cong(\mathcal{A}, \mathcal{H}, \bar{D})
$$

Since a change of Dirac operator in noncommutative geometry corresponds to a change in metric on the "manifold", the isomorphism (4.2) is simply the statement of general covariance of the noncommutative string spacetime.

A spacetime duality transformation is defined as an isomorphism of this type which identifies subspaces of the two spectral triples in (4.21) representing (classically) distinct ordinary spacetimes. The idea is represented symbolically by the diagram:

$$
\begin{array}{ccc}
(\mathcal{A}, \mathcal{H}, D) & \stackrel{T}{\longrightarrow} & (\mathcal{A}, \mathcal{H}, \bar{D}) \\
\Pi_{0} \downarrow & \downarrow \bar{\Pi}_{0} \\
\left(\mathcal{A}_{0}, \mathcal{H}_{0}, D_{0}\right) & \left(\overline{\mathcal{A}}_{0}, \overline{\mathcal{H}}_{0}, \bar{D}_{0}\right)
\end{array}
$$

In this diagram, the top line represents the isomorphisms between the full spectral triples, while $\Pi_{0}: \mathcal{H} \rightarrow \mathcal{H}_{0}$ and $\bar{\Pi}_{0}: \mathcal{H} \rightarrow \overline{\mathcal{H}}_{0}$ are orthogonal projections onto subspaces which represent classical spacetime geometries, e.g. $\left(\mathcal{A}_{0}, \mathcal{H}_{0}, D_{0}\right)=$ $\left(C^{\infty}\left(T_{d}, \mathbb{C}\right), L^{2}\left(T_{d}, S\right), i g^{i j} \gamma_{i} \partial_{j}\right)$ represents the ordinary torus $T_{d}$ with its flat metric $g_{i j}$. From the point of view of ordinary geometry, there is no reason for the two classical spacetimes in the bottom line of (4.3i) to be the same. However, their embeddings into the full spectral triple representing the noncommutative string spacetime defines an equivalence relation under the action of the unitary isomorphism $T$ which identifies them at the level of the quantum geometry, i.e. the mappings in (4.3) do not commute with the projection operators, and hence distinct classical spacetimes are identified. This is a very natural and powerful way to characterize string geometry in terms of different projections of the same spectral triple.

First, we shall establish that there indeed does exist a natural projection of the noncommutative string spacetime onto a classical, lowenergy sector. Consider the subspace

$$
\overline{\mathcal{H}}_{0}=\operatorname{ker} D \cong \bigotimes_{i=1}^{d}\left(\overline{\mathcal{H}}_{0}^{(+) i} \oplus \overline{\mathcal{H}}_{0}^{(-) i}\right)
$$

The states in $\overline{\mathcal{H}}_{0}$ are projected onto the vacuum sectors of the Fock spaces, i.e. their oscillatory parts vanish, as anticipated for a lowenergy regime of the string theory. The space $\overline{\mathcal{H}}_{0}^{(+) i}$ consists of those states which have vanishing momentum $p_{i}=0$ and whose spinor components carry the chiral action of the spin group defined by the action of the Dirac matrices as $g^{j k} d_{k i}^{+} \gamma_{j}^{+}=g^{j k} d_{k i}^{-} \gamma_{j}^{-}$(upon choosing appropriate boundary conditions for the spinors with respect to a homology basis of $\left.T_{d} \times T_{d}^{*}\right)$ in the $i$ th direction of the target space. On the other hand, $\overline{\mathcal{H}}_{0}^{(-) i}$ consists of those states which have zero winding number $w^{i}=0$ and which transform under the antichiral spinor representation defined by $\gamma_{i}^{+}=-\gamma_{i}^{-}$.

The $2^{d}$ subspaces in the decomposition (4.4) are all naturally isomorphic to one another [9in under "partial" $T$-duality transformations $\overline{\mathcal{H}}^{(\bar{\mp}) i} \leftrightarrow$ $\overline{\mathcal{H}}^{(-) i}$ for each $i$. This simply means that this decomposition is independent of the choice of spin structure on the torus (or equivalently of the choice of spinor boundary conditions along the elements of a homology basis), as it should be, and it is a remarkable fact that this independence is in itself an internal duality symmetry of the string theory. It suffices then to consider 
only the completely antichiral subspace

$$
\overline{\mathcal{H}}_{0}^{(-)}=\overline{\mathcal{H}}_{0}^{(-) 1} \otimes \overline{\mathcal{H}}_{0}^{(-) 2} \otimes \cdots \otimes \overline{\mathcal{H}}_{0}^{(-) d}
$$

which consists of those states of $\mathcal{H}$ which have the quantum representations $\gamma_{i}^{+}=-\gamma_{i}^{-} \equiv \gamma_{i}$ and $w^{i}=0$ for all $i=1, \ldots, d$. This is precisely what one expects for the low-energy (particlelike) regime of the string theory, whereby the chiral structure disappears and there are no nonlocal string modes that wind around the compactified directions of the target space. In fact, since $|p, p\rangle=\mathrm{e}^{-i p_{i} x^{i}}$, it follows that $\overline{\mathcal{H}}_{0}^{(-)} \cong$ $L^{2}\left(T_{d}, S^{-}\right)$, where $S^{-} \rightarrow T_{d}$ is the projection of the spin bundle $S \rightarrow T_{d} \times T_{d}^{*}$ onto its antichiral representation. It is also straightforward to see that the restriction of the antichiral Dirac operator to the subspace (4.

$$
\bar{D} \bar{\Pi}_{0}^{(-)}=i g^{i j} \gamma_{i} \frac{\partial}{\partial x^{j}}
$$

where $\bar{\Pi}_{0}^{(-)}: \mathcal{H} \rightarrow \overline{\mathcal{H}}_{0}^{(-)}$is the corresponding orthogonal projection. Finally, using the operatorstate correspondence, the corresponding algebra is taken to be the projection of the commutant of the chiral Dirac operator in $\mathcal{A}$ (the "restriction" of $\mathcal{A}$ to $(4.5 i))$,

$$
\overline{\mathcal{A}}_{0}^{(-)}=\bar{\Pi}_{0}^{(-)}\left(\operatorname{End}_{D} \mathcal{A}\right) \bar{\Pi}_{0}^{(-)}
$$

where $\operatorname{End}_{D} \mathcal{A}=\{V \in \mathcal{A} \mid[D, V]=0\}$ determines the largest subalgebra of $\mathcal{A}$ which acts densely on $(4.4)$. The elements of $(4.7)$ are those vertex operators which have no oscillatory modes and which create string states of identical left and right chiral momentum, i.e. $V_{q q} \sim \mathrm{e}^{-i q_{i} x^{i}}$, from which it follows that $\overline{\mathcal{A}}_{0}^{(-)} \cong C^{\infty}\left(T_{d}, \mathbb{C}\right)$. The construction described above thereby produces a subspace of the spectral triple $(\mathcal{A}, \mathcal{H}, \bar{D})$,

$$
\begin{aligned}
& \left(\overline{\mathcal{A}}_{0}^{(-)}, \overline{\mathcal{H}}_{0}^{(-)}, \bar{D} \bar{\Pi}_{0}^{(-)}\right) \\
& \quad \cong\left(C^{\infty}\left(T_{d}, \mathbb{C}\right), L^{2}\left(T_{d}, S^{-}\right), i g^{i j} \gamma_{i} \frac{\partial}{\partial x^{j}}\right)
\end{aligned}
$$

The spectral triple (4.81) represents the ordinary Riemannian geometry of the torus $T_{d}$ with metric $g_{i j}$, and thus the Dirac operator $D$ naturally defines the appropriate low-energy projection of the full noncommutative string spacetime via its zero-mode eigenspace ker $D \subset \mathcal{H}$ (or its dual version $\left.\operatorname{End}_{D} \mathcal{A} \subset \mathcal{A}\right)$.

The target space duality transformation (4.3i) follows from the observation that one could have chosen $\bar{D}$ instead of $D$ in the definition (4. 4 ) and carried out an analogous low energy projection. Doing so, we define

$$
\mathcal{H}_{0}=\operatorname{ker} \bar{D} \cong \bigotimes_{i=1}^{d}\left(\mathcal{H}_{0}^{(+) i} \oplus \mathcal{H}_{0}^{(-) i}\right)
$$

where $\mathcal{H}_{0}^{(+) i}$ consists of states with $\gamma_{i}^{+}=\gamma_{i}^{-}$and $w^{i}=0$, while the states of $\mathcal{H}_{0}^{(-) i}$ carry the quantum representation $g^{j k} d_{k i}^{+} \gamma_{j}^{+}=-g^{j k} d_{k i}^{-} \gamma_{j}^{-}$and $p_{i}=0$. Taking again the canonical choice

$$
\mathcal{H}_{0}^{(-)}=\mathcal{H}_{0}^{(-) 1} \otimes \mathcal{H}_{0}^{(-) 2} \otimes \cdots \otimes \mathcal{H}_{0}^{(-) d}
$$

in which $g^{j k} d_{k l}^{+} g^{l i} \gamma_{j}^{+}=-g^{j k} d_{k l}^{-} g^{l i} \gamma_{j}^{-} \equiv \gamma_{*}^{i}$ and $p_{i}=0$ for all $i=1, \ldots, d$, we find the isomorphism $\mathcal{H}_{0}^{(-)} \cong L^{2}\left(T_{d}^{*}, S^{-}\right)$under the identification $\left|d^{+} w,-d^{-} w\right\rangle \leftrightarrow \mathrm{e}^{-i w^{i} x_{i}^{*}}$. The projected chiral Dirac operator is

$$
D \Pi_{0}^{(-)}=i \gamma_{*}^{i} \frac{\partial}{\partial x_{i}^{*}}
$$

where $\Pi_{0}^{(-)}: \mathcal{H} \rightarrow \mathcal{H}_{0}^{(-)}$. Note that the Dirac matrices $\gamma_{*}^{i}$ generate the Clifford algebra with the dual metric

$$
\tilde{g}^{i j}=\left(d^{+}\right)^{i k} g_{k l}\left(d^{-}\right)^{l j}
$$

on $T_{d}^{*}$ which defines an inner product on the dual lattice $\Gamma^{*}$ (equal to the matrix inverse of $g_{i j}$ when $\beta=0)$. The low-energy subalgebra of $\mathcal{A}$ is now

$$
\mathcal{A}_{0}^{(-)}=\Pi_{0}^{(-)}\left(\operatorname{End}_{\bar{D}} \mathcal{A}\right) \Pi_{0}^{(-)}
$$

which consists of vertex operators of the form $V_{d^{+} v,-d^{-} v} \sim \mathrm{e}^{-i v^{i} x_{i}^{*}}$, so that $\mathcal{A}_{0}^{(-)} \cong C^{\infty}\left(T_{d}^{*}, \mathbb{C}\right)$. In this way we arrive at another low-energy commutative subspace

$$
\begin{aligned}
& \left(\mathcal{A}_{0}^{(-)}, \mathcal{H}_{0}^{(-)}, D \Pi_{0}^{(-)}\right) \\
& \quad \cong\left(C^{\infty}\left(T_{d}^{*}, \mathbb{C}\right), L^{2}\left(T_{d}^{*}, S^{-}\right), i \gamma_{*}^{i} \frac{\partial}{\partial x_{i}^{*}}\right)
\end{aligned}
$$

which represents the dual $d$-torus $T_{d}^{*}$ with metric $\tilde{g}^{i j}$.

From the point of view of classical general

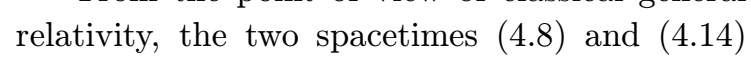


are inequivalent. However, consider the unitary transformation $T=T_{S} \otimes T_{X}: \mathcal{H} \rightarrow \mathcal{H}$ which acts as the gauge transformation

$$
\begin{aligned}
T_{X} & =\mathrm{e}^{i \mathcal{G}_{X}} \in \mathcal{U}(\mathcal{A}) \\
\mathcal{G}_{X} & =\frac{\pi}{2 i}\left(J_{+}^{+} J_{+}^{-}-J_{-}^{+} J_{-}^{-}\right) \\
J_{ \pm}^{a}\left(z_{a}\right) & =\stackrel{\circ}{\circ} \mathrm{e}^{ \pm i k_{i} X_{a}^{i}\left(z_{a}\right)} \circ
\end{aligned}
$$

where $a= \pm$ and $k_{i}$ is a Killing vector of $T_{d}$. It acts on the spectral data of the full noncommutative geometry as [9.9]

$$
\begin{aligned}
T_{X}\left|p^{+}, p^{-}\right\rangle= & (-1)^{p_{i} w^{i}} \\
& \times\left|\left(d^{+}\right)^{-1} p^{+},-\left(d^{-}\right)^{-1} p^{-}\right\rangle \\
T_{X} \alpha_{n}^{( \pm) i} T_{X}^{-1}= & \pm g_{j k}\left(d^{\mp}\right)^{i j} \alpha_{n}^{( \pm) k} \\
T_{S} \gamma_{i}^{ \pm} T_{S}^{-1}= & g^{j k} d_{i j}^{\mp} \gamma_{k}^{ \pm} \\
T_{X} V_{q^{+} q^{-}} T_{X}^{-1}= & (-1)^{q_{i} w^{i}} V_{q^{+}\left(d^{+}\right)^{-1},-q^{-}\left(d^{-}\right)^{-1}}
\end{aligned}
$$

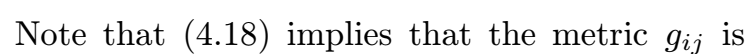
mapped to its dual ( $\bar{A} . \overline{1} \overline{2})$. It is straightforward to see that $T$ interchanges the two Dirac operators as prescribed in $(4 . \overline{1})$, and therefore gives the required isomorphism of the corresponding spectral triples. In this way, the distinct low energy classical spacetimes (4. $\left.\overline{8}^{i}\right)$ and $(\overline{\overline{1}} \overline{1} \overline{4})$ are identified, leading to the celebrated $T$-duality transformation of toroidally compactified string theory [i] We see therefore that in the framework of noncommutative geometry, the $T$-duality transformation of a toroidal target space is just the low energy projection of a gauge transformation on the noncommutative string spacetime.

The $T$-duality mapping actually determines only a $\mathbb{Z}_{2}$ subgroup of a larger discrete duality group of the string theory. The remaining generators come from the web of transformations between the other various subspaces of (1. $\left(\begin{array}{l}4 \\ 4\end{array}\right)$ and

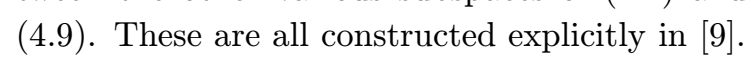
For example, the mapping between the antichiral subspace ('t. subspace $\mathcal{H}_{0}^{(+)^{-}}$determines the worldsheet parity symmetry of the string theory, which acts on the worldsheet by interchanging the chiral structures and on the background data as $\beta \rightarrow-\beta, d^{ \pm} \rightarrow$ $d^{\mp}$. Similarly, the mapping between $\left(4.5^{\top}\right)$ and the subspace of $\operatorname{ker} \bar{D}$ in which all tensor components have chiral conditions except for the $i$ th one leads to the factorized duality transformation of $T_{d}$, which can be thought of as the $R \rightarrow 1 / R$ circle duality along the $i$-th cycle of $T_{d}$. In the present framework, this transformation is also accompanied by a worldsheet parity map in all of the other $d-1$ directions. When $d$ is even, the factorized dualities lead to the phenomenon of mirror symmetry (and hence of spacetime topology change) which exchanges the complex and Kähler structures of the torus. In addition there other dualities which act trivially on the spectral triples, but nonetheless do lead to non-trivial quantum dynamics and are therefore considered as symmetries of the quantum geometry. These are the changes of basis of the compactification lattice $\Gamma$, and the torsion cohomology shift $\beta_{i j} \rightarrow \beta_{i j}+\lambda_{i j}$, with $\lambda_{i j}$ an antisymmetric integer-valued matrix, which can be absorbed into a redefinition of the momenta $p_{i} \rightarrow p_{i}-$ $\lambda_{i j} w^{j}$. Altogether these transformations generate the discrete duality group which is the semidirect product

$$
G_{d}=O(d, d ; \mathbb{Z}) \otimes \mathbb{Z}_{2}
$$

of the isometry group $\operatorname{Aut}\left(\Gamma^{*} \oplus \Gamma\right)=O(d, d ; \mathbb{Z})$ by the natural action of the worldsheet parity group $\mathbb{Z}_{2}$. The duality group ('A. set of low energy theories and connects various inequivalent classical spacetimes. Its action also leaves the spectra of the Dirac operators $D$ and $\bar{D}$ invariant.

\section{Gauge Symmetries}

In the previous section we saw that the duality group $(\bar{A} . \overline{0} \overline{0})$ of toroidally compactified string theory arises in a very natural way through the automorphisms of the noncommutative string spacetime. With the exception of worldsheet parity, the transformations we described above were all realized as inner automorphisms and so represented gauge symmetries of the noncommutative geometry [9.9. On the other hand, worldsheet parity exchanges the left and right chiral algebras $\mathcal{E}^{ \pm} \rightarrow \mathcal{E}^{\mp}$ and therefore represents an outer automorphism of the vertex operator algebra (since 
no element of $\mathcal{A}$ can accomplish this chirality reversal). In this final section we shall study in more depth the automorphism group of the vertex operator algebra, which represents the homeomorphisms of the noncommutative space, and focus on the consequences of the realization of duality transformations as gauge symmetries. Here we shall set $\beta_{i j}=0$.

\section{Automorphisms of Lattice Vertex Opera- tor Algebras}

Generally, the inner automorphisms of a $*$-algebra $\mathcal{A}$ form a normal subgroup $\operatorname{Inn}(\mathcal{A})$ of the automorphism group $\operatorname{Aut}(\mathcal{A})$. The remaining symmetries $\operatorname{Out}(\mathcal{A})=\operatorname{Aut}(\mathcal{A}) / \operatorname{Inn}(\mathcal{A})$ form the outer automorphisms of the algebra such that the automorphism group is the semi-direct product

$$
\operatorname{Aut}(\mathcal{A})=\operatorname{Inn}(\mathcal{A}) \otimes \operatorname{Out}(\mathcal{A})
$$

of $\operatorname{Inn}(\mathcal{A})$ by the natural action of $\operatorname{Out}(\mathcal{A})$. The inner automorphisms represent internal fluctuations of the noncommutative geometry corre-

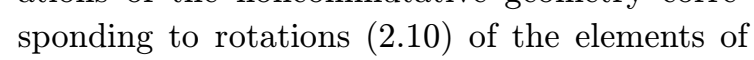
$\mathcal{A}$. Consider the example of a spin manifold discussed in section 2. In this case the inner automorphisms of $\mathcal{A}=C^{\infty}(M, \mathbb{C})$ are all trivial (there are no internal symmetries for a commutative algebra), while $\operatorname{Out}(\mathcal{A}) \cong \operatorname{Diff}(M)$ (given a diffeomorphism $\phi \in \operatorname{Diff}(M)$, one may construct the natural automorphism $g_{\phi}(f)=f \circ \phi^{-1} \quad \forall f \in$ $\mathcal{A})$. The outer automorphisms in this case generate the general covariance of the space. On the other hand, for the case $\mathcal{A}=C^{\infty}(M, \mathbb{C}) \otimes$ $M_{N}(\mathbb{C})$, while the outer automorphisms still generate general coordinate transformations of the manifold $M$, the inner automorphisms generate the group of $U(N)$ gauge transformations on $M$.

In these two examples there is a clear distinction between (internal) gauge symmetries and outer automorphisms, again because there is an underlying commutative algebra which is simply augmented by a discrete internal space. This is not the case of the string theory spectral triple, which represents a genuine noncommutative space. As a dramatic example, we recall that the conserved currents ( $(3 . \overline{2} \overline{1} \overline{1})$ are the generators of target space reparametrizations, so that a general coordinate transformation $X \rightarrow \xi(X)$ of the space- time may be represented by the gauge transformation [9, $[\overline{9}]$

$$
\begin{aligned}
& T_{\xi}=\mathrm{e}^{i \mathcal{G}_{\xi}} \in \mathcal{U}(\mathcal{A}) \\
& \mathcal{G}_{\xi}=\xi_{i}(X)\left(\delta_{+}^{i}+\delta_{-}^{i}\right)
\end{aligned}
$$

However, these inner automorphisms are mapped onto outer automorphisms of the commutative algebra $\overline{\mathcal{A}}_{0}^{(-)} \cong C^{\infty}\left(T_{d}, \mathbb{C}\right)$, representing the diffeomorphisms of the torus, under the low energy projection onto the ordinary geometry of $T_{d}$. This is a very nontrivial fact, as it implies that general covariance is manifested as a gauge symmetry of the full noncommutative string spacetime, and in this sense general relativity is in fact really a gauge theory. This is of course what one would like of a theory that unifies all of the fundamental interactions, in that it puts gauge and gravitational interactions on the same footing. Note that in this sense the worldsheet parity transformations are implemented as "diffeomorphisms" of the noncommutative string spacetime.

The realization of dualities as gauge symmetries yields a natural explanation of the long standing puzzle as to the origin of string duality as part of some mysterious gauge group [i, In the present case, the conserved currents $(3.2 \overline{1})$ along with the chiral tachyon vertex operators generate the affine Lie group $\widehat{\operatorname{Inn}}^{(0)}(\mathcal{A})$ of primary fields of weight 1 [12 2in. The corresponding inner automorphisms give internal perturbations which preserve the grading by conformal dimension and thereby yield isomorphic conformal field theories. Generically, this group is just the $U(1)_{+}^{d} \times U(1)_{-}^{d}$ Kac-Moody group, but there are points in the moduli space of toroidal compactifications at which this group is enhanced to a non-abelian gauge symmetry, such as an affine $S U(2)_{+}^{d} \times S U(2)_{-}^{d}$ Kac-Moody group. A natural subgroup of the automorphism group of a lattice vertex operator algebra is therefore given by [1] $[1]$

$$
\operatorname{Aut}^{(0)}(\mathcal{A})=\widehat{\operatorname{Inn}}^{(0)}(\mathcal{A}) \otimes \operatorname{Out}(\mathcal{A})
$$

where the group of outer automorphisms is

$$
\operatorname{Out}(\mathcal{A}) \cong O(d, d ; \mathbb{Z}) \otimes O(2 ; \mathbb{R})
$$

where $O(d, d ; \mathbb{Z})$ represents those automorphisms which preserve the bilinear form of the Lorentzian 
lattice $\Gamma^{*} \oplus \Gamma$, while $O(2 ; \mathbb{R})$ is a worldsheet symmetry group which is an extension of worldsheet parity that acts by rotating the two chiral sectors of the theory among each other. The full automorphism group is very large and is not known in explicit form. It is related to some exotic mathematical constructions, such as the Monster sporadic group $\left[\begin{array}{l}1 \\ 1\end{array}\right.$ and the occurence of duality as a gauge symmetry is a generic property of all duality symmetries. For instance, all of these structures can be shown to hold for asymmetric duality groups, such as those associated with heterotic strings [14], and also for non-perturbative $S L(2 ; \mathbb{Z}) S$ duality symmetries [1 $\left.11^{1}\right]$.

\section{Duality Equivalence Classes}

In the previous section we saw the effects of quantum geometry at the level of classical spectral triples. The full-blown noncommutative geometry comes into play at the level of the automorphisms which implement these quantum symmetries. For instance, the diffeomorphism gauge symmetries $(\overline{5} \cdot \overline{2})$ are associated with the graviton operators $(13 . \overline{2} \overline{0})$ which represent the lowest oscillatory excitation of the classical spacetime. The other set of generators of $\widehat{\operatorname{Inn}}^{(0)}(\mathcal{A})$, i.e. the tachyon vertex operators, turn out to also give a noncommutative perturbation of classical spacetime that we shall now proceed to describe.

The operator product of two tachyon operators may be written as

$$
\begin{aligned}
& V_{q^{+} q^{-}}\left(z_{+}, z_{-}\right) V_{r^{+} r^{-}}\left(w_{+}, w_{-}\right) \\
& =\left(z_{+}-w_{+}\right)^{q_{i}^{+} g^{i j} r_{j}^{+}}\left(z_{-}-w_{-}\right)^{q_{i}^{-} g^{i j} r_{j}^{-}} \\
& \quad \times \quad{ }_{\circ}^{\circ} V_{q^{+} q^{-}}\left(z_{+}, z_{-}\right) V_{r^{+} r^{-}}\left(w_{+}, w_{-}\right) \stackrel{\circ}{\circ}
\end{aligned}
$$

The expression $\left(\begin{array}{c}-\overline{5} . \overline{5} \\ )\end{array}\right)$ is in general singular at coinciding points of the operators, and to make proper sense of it the vertex operators must be suitably regularized. Doing so (see [1] $\left.{ }^{1} 3_{1}^{1}\right]$ for details), by interchanging the order of the two operators the relation (5.5) induces a local cocycle relation which, up to a permutation of the coordinate directions of $T_{d} \times T_{d}^{*}$, is independent of the worldsheet coordinates $z_{ \pm}$and $w_{ \pm}$. This local cocycle relation may be easily recognized as the defining relation of the noncommutative torus $\left(\overline{2} . \overline{1} \overline{6}_{1}^{i}\right)$ with the generators $U_{i}$ identified with the tachyon operators $V_{e_{+}^{i} e_{-}^{i}}$, where $e_{ \pm}^{i}$ is the canonical basis of the Lorentzian lattice $\Gamma^{*} \oplus \Gamma$. The deformation parameter is related to the metric of $T_{d}$ by [1] $\left.\overline{3}_{1}^{\prime}\right]$

$$
\omega^{i j}=\operatorname{sgn}(j-i) g^{i j} \quad, \quad i \neq j
$$

More precisely, the tachyon algebra generated by the operators $V_{q^{+} q^{-}}$defines a unitary equivalence class of self-dual $\mathbb{Z}_{2}$-twisted projective modules of rank $2 d$ over the double noncommutative torus $T_{\omega}^{(+) d} \times T_{\omega}^{(-) d}$ (one copy for each chiral sector of the vertex operator algebra along with a $\mathbb{Z}_{2}$ twist coming from the cocycle factors in $\left.\left(3 . \overline{1} \overline{7}^{-}\right)\right)$. Notice, however, that according to (13.18) the product of two projected tachyon operators (made via the operator-state correspondence by mapping onto $\left.L^{2}\left(T_{d} \times T_{d}^{*}, S\right)\right)$ is commutative and just represents the algebra of functions on $T_{d} \times T_{d}^{*}$. In the case at hand, two given operators are first multiplied together in the full algebra, and then the result is projected onto the tachyon sector of the noncommutative geometry. Namely, given $V_{0}=\Pi_{0} V \Pi_{0}$ and $W_{0}=\Pi_{0} W \Pi_{0}$, where $V, W \in$ $\mathcal{A}$ and $\Pi_{0}: \mathcal{H} \rightarrow L^{2}\left(T_{d} \times T_{d}^{*}, S\right)$, the deformed product is given by

$$
V_{0} \star_{\omega} W_{0}=\Pi_{0}(V W) \Pi_{0}
$$

Thus the algebraic properties of the vertex operator algebra give a very natural way to deform the algebra of functions on $T_{d} \times T_{d}^{*}$ which takes into account of the internal oscillatory modes of the strings.

We see therefore that the intermediate regime separating the classical torus from the highly noncommutative string spacetime with its oscillators turned on is the tachyon algebra which is a module for the noncommutative torus. Note that for very large distance scales, i.e. $g_{i j} \rightarrow$ $\infty$, the deformation parameter $(\underline{-5} \cdot \overline{6})$ vanishes and the tachyon algebra coincides with the algebra of functions on the double torus. This is just the statement that at very large distance scales we recover a classical spacetime. On the other hand, at very small distance scales $g_{i j} \rightarrow 0$ and thus, within the framework of toroidally compactified string theory, spacetime at very short distances is a noncommutative torus. 
Having identified this intermediate regime allows us to explore features of the noncommutative geometry without having to deal with all of the complicated structures that make up the full vertex operator algebra. Moreover, it enables us to put the notion of string duality into a more familiar mathematical parlance. Namely, the deformation parameter $\left(\bar{b}^{-} . \overline{\sigma_{i}}\right)$ is the natural induced antisymmetric bilinear form on the lattice $\Gamma$, and so it transforms under the action of the target space duality group $O(d, d ; \mathbb{Z})$ as in $(12 . \overline{2} \overline{2})$ (2) Each such orbit is implemented in the full algebra $\mathcal{A}$ by a gauge transformation $T$ as described above. Under the equivalence relation generated by the isomorphism of full spectral triples, the modules for the noncommutative tori with deformation parameters $\omega$ and $\omega^{*}$ are identified. In other words, target space dualities are naturally realized as the Morita equivalences among noncommutative tori. This remarkable feature of string geometry can be thought of as lending a physical interpretation to the mathematical notion of Morita equivalence in noncommutative geometry. It is also in accord with the connections that exist between the noncommutative torus and Matrix Theory [i]n], in which the deformation parameter $\omega$ is given by the light-like component of the three-form tensor field coming from the corresponding reduction of 11-dimensional supergravity. In that case Morita equivalence relates dual quantum theories which have the same BPS spectra [222].

\section{Universal Gauge Symmetry}

Let us now take the Lorentzian lattice to be $\Gamma^{*} \oplus$ $\Gamma=\mathbb{Z} \oplus \omega \mathbb{Z}$ and assume that the deformation parameter is a rational number

$$
\omega=M / N
$$

where $M$ and $N$ are relatively prime positive integers. The center of $\mathcal{A}^{(\omega)}$

$$
\mathcal{Z}\left(\mathcal{A}^{(\omega)}\right)=\operatorname{span}_{\mathbb{C}}\left\{U_{1}^{m N} U_{2}^{n N} \mid m, n \in \mathbb{Z}\right\}
$$

is infinite dimensional, and the quotient $\hat{\mathcal{A}}^{(\omega)}=$ $\mathcal{A}^{(\omega)} / \mathcal{I}_{\omega}$ by the ideal $\mathcal{I}_{\omega}$ generated by $\mathcal{Z}\left(\mathcal{A}^{(\omega)}\right)-$ $\{\mathbb{I}\}$ is isomorphic, as a unital $*$-algebra, to $M_{N}(\mathbb{C})$. The clock algebra $U_{1} U_{2}=\mathrm{e}^{2 \pi i \omega} U_{2} U_{1}$ can be rep- resented by the $N \times N$ clock and shift matrices

$$
\begin{aligned}
U_{1}= & \left(\begin{array}{ccccc}
1 & 0 & 0 & \ldots & 0 \\
0 & q & 0 & \ldots & 0 \\
0 & 0 & q^{2} & \ldots & 0 \\
\vdots & \vdots & \vdots & \ddots & \vdots \\
0 & 0 & 0 & \ldots & q^{N-1}
\end{array}\right) \\
U_{2}= & \left(\begin{array}{ccccc}
0 & 1 & 0 & \ldots & 0 \\
0 & 0 & 1 & \ldots & 0 \\
\vdots & \vdots & \vdots & \ddots & \vdots \\
0 & 0 & 0 & \ldots & 1 \\
1 & 0 & 0 & \ldots & 0
\end{array}\right)
\end{aligned}
$$

where $q=\mathrm{e}^{2 \pi i M / N}$.

The physical relevance of this special case is many-fold. First of all, any "smooth" element of the $s u(N)$ Lie algebra can be expanded in terms of products of the generators $(\overline{5} . \overline{1} \overline{0})$, such that in the limit $N \rightarrow \infty, \omega \rightarrow 0$ it becomes the Poisson-Lie algebra of smooth functions on the 2-torus with respect to the usual Poisson bracket

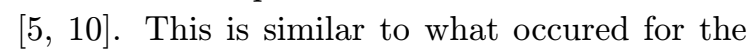
vertex operator algebra, whereby some of the gauge symmetries of the noncommutative geometry projected onto outer automorphisms of the classical spacetime. The large- $N$ limit of $S U(N)$ is relevant to the Matrix Theory description of M-theory, in which $N$ is proportional to the longitudinal momentum and the limit $N \rightarrow \infty$ describes dynamics in the infinite momentum frame $\left[\bar{i}_{1}\right]$. The gauge symmetry here is then represented by the infinite unitary group $S U(\infty)$ which is an

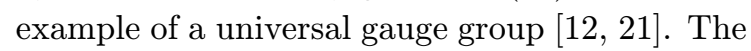
canonical universal gauge groups of vertex operator algebras, representing the algebras overlying all of the dynamical symmetries of string theory, may thus be related, through the connection with the noncommutative torus, to $S U(\infty)$.

More ambitiously, one can imagine that the noncommutative 2-torus with rational deformation parameter (5.8i) is some sort of finite dimensional approximation to the vertex operator algebra. In the correlated limit $N, M \rightarrow \infty$ with $\omega$ a fixed irrational number, we recover the genuine noncommutativetorus (the center (15.9i) is then trivial) which is related to a corresponding vertex operator algebra as described above with an explicit representation of the universal gauge symmetry. The noncommutative geometry asso- 
ciated with rank 2 lattice vertex operator algebras may in this way be relevant to the true noncommutative geometric structure encompassing the large- $N$ limit of Matrix Theory. However, in order for this to be true, one needs to realize the noncommutative string spacetime as some limit of a tower of finite dimensional matrix geometries.

The potential relevance to Matrix Theory compactifications comes from the observation that the Morita equivalence of the noncommutative 2 -torus can be naturally interpreted as the zero area limit of the usual string theoretical $S L(2 ; \mathbb{Z})$ duality in the presence of a background NeveuSchwarz two-form field $\beta\left[23^{3}\right.$. In supergravity, this is not a geometrical symmetry in the usual sense, but in the framework of noncommutative geometry it is. In the present case, the field $\beta$ is given explicitly by (5.6) and in the zero area limit we obtain a highly noncommutative structure. This is in precise agreement with the idea that toroidal compactifications at very small distance scales are equivalent to noncommutative tori.

\section{Noncommutative Gauge Theory}

The mapping onto the noncommutative torus can also be used to give a dynamical model for the duality symmetries of the noncommutative string spacetime, through the construction of an explicitly duality symmetric noncommutative gauge theory $13 \overline{1}$. The action functional of the gauge theory defined from the twisted module over the noncommutative torus is obtained using the in-

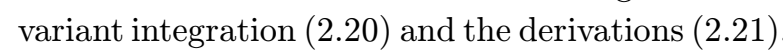
The interaction vertices are constructed from the commutator which is represented by the Moyal bracket

$$
[f, g]=\{f, g\}_{\omega}=f \star_{\omega} g-g \star_{\omega} f
$$

where the deformed product of two functions $f, g \in$ $C^{\infty}\left(T_{d} \times T_{d}^{*}, \mathbb{C}\right)$ is given by

$$
\begin{aligned}
& \left(f \star_{\omega} g\right)\left(x, x^{*}\right) \\
& =\exp \left[i \pi \omega^{i j}\left(\frac{\partial}{\partial x^{i}} \frac{\partial}{\partial x^{\prime j}}-g_{i k} g_{j l} \frac{\partial}{\partial x_{k}^{*}} \frac{\partial}{\partial x_{l}^{\prime *}}\right)\right] \\
& \quad \times\left. f\left(x, x^{*}\right) g\left(x^{\prime}, x^{\prime *}\right)\right|_{\left(x^{\prime}, x^{\prime *}\right)=\left(x, x^{*}\right)}
\end{aligned}
$$

Using the projections of the two Dirac operators $D$ and $\bar{D}$ introduced earlier onto the tachyon algebra, one may naturally write down bosonic and fermionic Lagrangians in the usual spirit of noncommutative geometry [1] 13 in],

$$
\begin{aligned}
& \mathcal{L}=\left(F+{ }^{\star} F\right)_{i j}\left(F+{ }^{\star} F\right)^{i j} \\
& -i \overline{\psi_{*}} \gamma^{i}\left(\partial_{i}+i \overleftrightarrow{A_{i}}\right) \psi-i \bar{\psi} \gamma_{i}^{*}\left(\partial_{*}^{i}+i \stackrel{\leftrightarrow}{A_{*}^{i}}\right) \psi_{*}
\end{aligned}
$$

The bosonic part yields a symmetrized YangMills type functional with the above interaction vertices, constructed from two vector potentials $A\left(x, x^{*}\right)$ and $A_{*}\left(x, x^{*}\right)$. The fermionic part contains fermion fields minimally coupled to the gauge potentials, again determined by a non-local interaction vertex coming from the representation of the noncommutative tachyon algebra on the Hilbert space $L^{2}\left(T_{d} \times T_{d}^{*}, S\right)$, i.e.

$$
\begin{aligned}
\left(V_{q^{+} q^{-}} f\right)_{r^{+} r^{-}}= & (-1)^{q_{i} v^{i}} \mathrm{e}^{2 \pi i\left(q_{i}^{+} g^{i j} r_{j}^{+}+q_{i}^{-} g^{i j} r_{j}^{-}\right)} \\
& \times f_{q^{+}+r^{+}, q^{-}+r^{-}}
\end{aligned}
$$

for $f \in \mathcal{S}\left(\Gamma^{*} \oplus \Gamma\right)$.

The noncommutative gauge theory so constructed in possesses a number of symmetries. The fact that the action is manifestly gauge invariant leads immediately to its explicit invariance under target space duality transformations (represented by the actions of inner automorphisms) and also its general covariance. It is also invariant under the outer automorphism group (5.) representing "diffeomorphisms" of the noncommutative geometry. This includes the Morita equivalence acting on the doublet of vector potentials by the vector representation of $O(d, d ; \mathbb{Z})$, and also the $O(2 ; \mathbb{R})$ chiral rotations of the doublet. Like the usual formulations of duality symmetric action functionals, this model possesses an $O(2 ; \mathbb{R})$ doublet of vector potentials, but the present gauge theory is generally covariant without the need of introducing auxiliary fields. The price to pay for this is the nonlocality of the gauge interactions, but from the point of view of quantum field theoretic renormalizability the present model is in fact completely well-defined. The gauge theory can be considered as a dynamical model which measures how much duality symmetry is present in the target space and 
hence how far away the stringy perturbation is from ordinary_classical spacetime [1 131 . The act tion functional can thereby be regarded_as an [9] F. Lizzi and R.J. Szabo, 'Phys. Rev. Lett. 79 effective measure of distance scales in the space-

time. (1997) 3581'; 'Comm. Math. Phys. 197 (1998)' $66 \underline{7}^{-}$

[10] J. Fröhlich, O. Grandjean and A. Recknagel, Les Houches Session LXIV (North-Holland, 1998).

The nonlocality which arises in this field theory can also be shown to be induced by $\bar{D} \overline{1}=$ branes wrapping a highly oblique 2-torus [23] whose worldvolume field theory is thereby described by $2+1$ dimensional noncommutative gauge theory. By $T$-duality, this gauge theory also describes D0-branes on a very small 2-torus with non-zero Neveu-Schwarz two-form field, which in the large $N$ limit becomes the Matrix Theory description of M-theory with a non-zero background three-form field and a light-like compactification circle. It would be interesting to pursue further the interrelationships that exist between the two noncommutative geometric approaches, one based_on (worldsheet) vertex operator algebras and the other on (target space) Matrix Theory compactifications, to string theory and Mtheory. Their very natural relationships to the noncommutative torus hints that the connection may not be very far away.

\section{References}

[1] G. Veneziano, Europhys. Lett. 2 (1986) 199; D.J. Gross and P.F. Mende, iNucl. Phys. B $\mathbf{3 0 3}$ (1988) 407

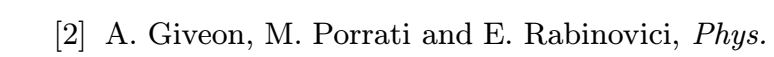
-

[3] A. Connes, Noncommutative Geometry (Academic Press, 1994); J. Madore, An Introduction to Noncommutative Geometry and its Physical Applications, LMS Lecture Notes 206 (1995);

, - - - G. Landi,_An_Introduction _to Noncommutative

' - - _ Spaces and _tbeir_Ceometries_(Springer-Verlag, 1997).

[11] F. Lizzi and R.J. Szabo, 'Phys. Lett. B _.417! - 1998$)$ 303.

[12] F. Lizzi and R.J. Szabo, Chaos, Solitons and Fractals 10 (1999) 445.

[13] G. Landi, F. Lizzi and R.J. Szabo, hep-th/9806099, to appear in Comm. Math. Phys.

[14] D.D. Song and R.J. Szabo, hep-th/9812235.'

[15] F. Lizzi, hep-th/9902187!

[16] J.M.G. Fell and R.S. Doran, Representations of *-algebras, Locally Compact Groups and Banach *-algebraic Bundles (Academic Press, 1988).

[17] A. Connes and J. Lott, 'Nucl. Phys. 18B (Proc.' Suppl.)_(1990)_29; A. Connes, J. Math. Phys. 36 (1995) 619.C.P. Martín, J.M. Gracia-Bondía and J.C. Várilly, 'Ph

[18] M.A. Rieffel and A.S. Schwarz, 'q-alg/9803057', to appear in Int. J. Math.

[19] I.B. Frenkel, J. Lepowsky and A. Meurman, Vertex Operator Algebras and the Monster, Pure Appl. Math. 134 (Academic Press, 1988); R.W. Gebert, Int. J. Mod. Phys. A 8 (1993) 5441; V.G. Kac, Vertex Algebras for Beginners, AMS University Lecture Series 10 (1997).

[20] E. Witten, J. Diff. Geom. 17 (1982) 661; 'ㅎuc.'.' Phys. B 202 (1982) 253'.

[21] S.G. Rajeev, Phys. Rev. D42 (1990) 2779; D44 (1991) 1836; C.-W.H. Lee and S.G. Rajeev, hep-th/9712090.

[22] A.S. Schwarz, 'Nucl. Phys. B 534 (1998) 720.

[23] M.R. Douglas and C.M. Hull, 'J. High Energy Phys. 02 (1998) 008; Y.-K.E. Cheung and M. Krogh, 'Nucl. Phys. B $\mathbf{5 2 8}(1998)$ 185.

[4] E. Witten, 'Nucl. Phys. B 460 (1996) 335!'

[5] T. Banks, W. Fischler, S.H. Shenker and L. Susskind, Phys. Rev. D $\mathbf{5} 5(1997) 5112$.

[6] A. Connes, M.R. Douglas and A.S. Schwarz, 'J.' ' - _ High Energy Phys. 02 (1998) 003'.

[7] J. Fröhlich and K. Gawędzki, CRM Proc. Lecture Notes 7 (1994) 57.

[8] A.H. Chamseddine, 'Phys. Lett. B 400 (1997)!',

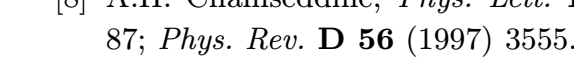

